\section{Coloboma of Iris and Choroid: Two Case Reports}

\author{
Min Sun ${ }^{1,2}$, Dan Wei ${ }^{3}$, Hui lin ${ }^{4}$ and Jian Ye $\mathrm{e}^{1,2^{*}}$ \\ ${ }^{1}$ Department of Ophthalmology, Daping Hospital, Chongqing, PR China \\ ${ }^{2}$ Research Institute of Surgery, Third Military Medicine University, Chongq- \\ ing, PR China
}

${ }^{3}$ Department of Ophthalmology, Yongchuan Traditional Chinese Medicine Hospital, Chongqing, PR China

${ }^{4}$ Department of Ophthalmology, The Chinese People's Liberation Army 252 hospital, Hebei Province, PR China

\begin{abstract}
Ocular coloboma, generally classified as typical and atypical, is a rare malformation occurred as an isolated defect in healthy individuals or a part of a complex malformation syndrome. In typical ones, iris coloboma and choroid coloboma often exist concomitantly and are associated with early cataractous changes. In this report, we described three eyes of two patients with coloboma of both iris and choroidal associated with complicated cataract. The patients suffered a low vision after the complicated cataract, surgery of phacoemulsification and intraocular lens implantation was performed on one eye in both patients. The Best Corrected Visual Activity (BCVA) of the eyes received cataract surgery improved greatly.
\end{abstract}

\section{Introduction}

Ocular coloboma is a rare malformation which occurs as an isolated defect in healthy individuals or be part of a complex malformation syndrome of known or unknown etiology [1-3]. Coloboma is generally classified as typical or atypical, depending on their location in the iris or fundus.

Typical iris coloboma is usually assumed as a complete thickness defect of the iris stroma and pigment epithelium which extends inferonasally to the corneoscleral limbus. Therefore, pear-shaped pupil is the characteristic change in typical iris coloboma. Typical chorioretinal coloboma, often affecting both eyes, is usually glistening white defects with distinct margins often rimmed by irregular pigment clumps. Typical iris and choroid coloboma often exist concomitantly and are

*Corresponding author: Jian Ye, Department of Ophthalmology, Daping Hospital; Research Institute of Surgery, Third Military Medicine University; Changjiang Branch Road, Chongqing, PR China, Tel: +86 2368767066; E-mail: yejian1979@163.com

Citation: Sun M, Wei D, Lin H, Ye J (2017) Coloboma of Iris and Choroid: Two Case Reports. J Ophthalmic Clin Res 4: 034.

Received: August 17, 2017; Accepted: September 27, 2017; Published: October 12, 2017 associated with congenital microphthalmos or/and optic nerve Coloboma [1]. It is reported that chorioretinal coloboma occurs in $0.14 \%$ of the general population, and $40 \%$ of affected individuals may develop retinal detachment sometime during their lifetime [4,5].

We described three eyes of two patients with coloboma of both iris and choroidal associated with complicated cataract. Surgery of phacoemulsification and intraocular lens implantation was performed on one eye of both patients and the Best Corrected Visual Acuity (BCVA) of the eyes which have a surgery improved greatly.

\section{Case 1}

A 40-year old Chinese man sought treatment for the progressive deterioration of vision in the right eye over about five months period. His sister also had congenital ocular abnormalities. Because of some reasons, the detailed examinations on his sister were not available.

On examination, the Snellen visual acuity of the right eye and left eye was 0.15 and 0.6 , respectively. The intraocular pressure of the right eye and the left eye were 11.3 and $12.7 \mathrm{mmHg}$, respectively. Slitl amp examination showed clear corneas in both eyes. Obviously, the patient had a bilateral inferonasal coloboma of iris, which resulted in the pear-shaped pupil in both eyes (Figure 1). The remarkable opaqueness of lens in the right eye, which was much severe than that in the left eye, may result in the lower visual acuity.
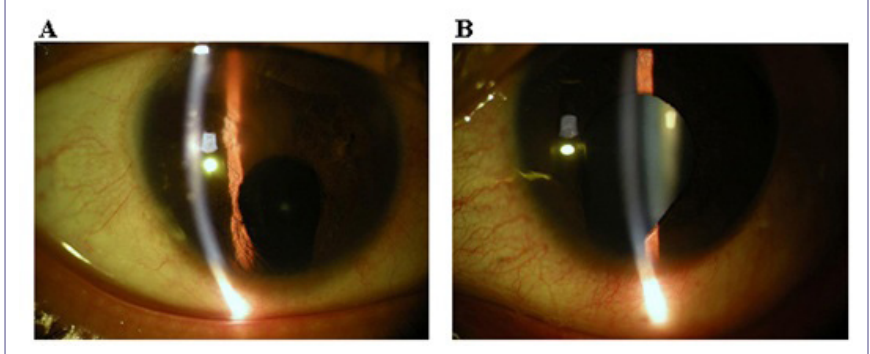

Figure 1: A) Anterior segment photography of the right eye of the first patient after cataract surgery: the right eye of the patient had an inferonasal coloboma of iris, which resulted in the pear-shaped pupil in this eye. B) Anterior segment photography of the left eye of the first patient before surgery: the left eye of the patient had an inferonasal coloboma of iris, which resulted in the pear-shaped pupil in this eye.

Fundus examination revealed bilateral inferior choroidal coloboma under the optic disc, which was about five disc areas in the right eye and about three disc areas in the left eye, respectively (Figure 2). Fortunately, the macular lutea of the retina in both two eyes were not affected. No retinal break was visible in the area of the coloboma or in the periphery. No obvious retinal detachment was observed in both eyes.

The results of Ultrasound Biomicroscopy (UBM) showed that, in both eyes, the inferior angles were damaged. To a varying degree, the angles in the rest quadrants were back. The bottom parts of the iris missed partially. Importantly, the echoes of inferior part of ciliary body showed abnormalities. However, the ciliary body in the other 
quadrants was exist and normal. The results of electrophysiology in both eyes failed to find any abnormality. In addition, the patients already had concomitant external strabismus when he referred to our clinic.

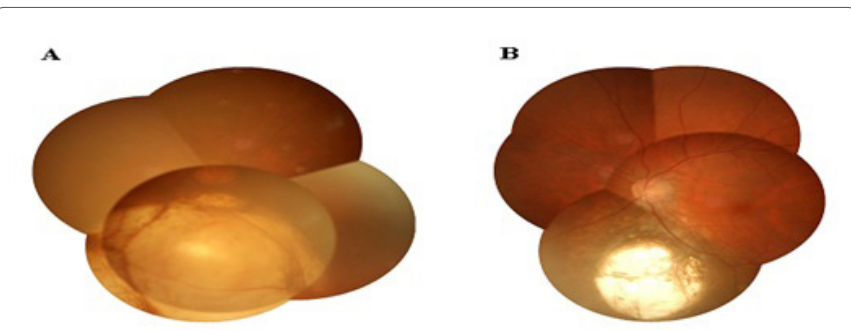

Figure 2: A) Fundus photography of the first patient's right eye before surgery: the picture shows about five disc areas' inferior choroidal coloboma under the optic disc. B) Fundus photography of the first patient's left eye before surgery: the picture shows about three disc areas' inferior choroidal coloboma under the optic disc.

Because of the severe lens opacity in the right eye and the low visual acuity, the patient accepted the suggestion of cataract surgery for this right eye. Consequently, phacoemulsification and intraocular lens implantation were performed on the right eye. Three days after surgery, the visual acuity of the right eye was 0.6 and remained on this level until now (18 months).

\section{Case 2}

Another patient was a 45 -year old male Chinese. The visual acuity of the left eye was decreased gradually for three years. On examination, the Snellen visual acuity of the left eye was 0.1 . The intraocular pressure of the left eye was $17.9 \mathrm{mmHg}$. Slit lamp showed clear corneas in the left eye. The patient had an obvious inferonasal coloboma of iris, which resulted in the pear-shaped pupil in left eye. The lens in the left eye was severely opaque, which may result in the low visual acuity. Fundus examination revealed inferior choroidal coloboma of five disc diameter in the left eye (Figure 3). The macular lutea of the retina in the left eye was not affected. No retinal break was identified and no obvious retinal detachment presented in the left eye. The visual acuity of right eye had been no light perception for more than twenty years. The cornea of the right eye was muddy and we could not identify the intraocular structure. Therefore, we could not determine whether the right eye had coloboma of iris and choroid or not.

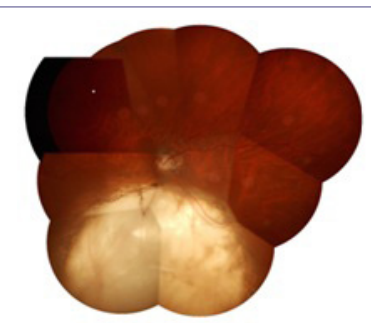

Figure 3: Fundus photography of the second patient's left eye after surgery: fundus examination revealed inferior choroidal coloboma of five disc diameter in the left eye.

Because of the severe lens opacity in the left eye, cataract surgery was recommended. Phacoemulsification and intraocular lens implantation was performed on the eye. Two days after surgery, the visual acuity of this eye was 0.8 and remains good up to now (16 months).

\section{Discussion}

In this study, we described two patients with three eyes affected by coloboma of iris and choroid. Concurrently, three eyes of these two patients had complicated cataract. No obvious retinal detachment was observed in these three eyes. The macular lutea in these three eyes were not attacked concomitantly. Cataract surgery was performed on two eyes and the BCVA after operation improved significantly.

It is reported that flattening of the exposed portion of the lens, gaps in the zonular fibers and localized cortical lens opacities or pigment clumps on the lens capsule at the exposed margin often accompany iris coloboma [6,7]. Visually significant cataracts do occur, but are rare. The right eye's lens of the first patient and the left eye's lens of the second patient were severely cloudy and the BCVA in these two eyes decreased significantly. Meanwhile, the left eye's lens of the first patient was cloudy slightly and the BCVA in this eye remains relatively normal. However, there were no obvious malformations in all three lenses we reported. Gopal et al., reviewed 85 eyes of 81 patients with retinal detachments related to coloboma of the choroid [8]. According to the report, retinal detachment could occur in eyes with choroidal coloboma because of a retinal break outside the colobomatous area, at the margin of the coloboma, or over the coloboma [8]. It has been reported that persistent fetal vasculature and fundal coloboma are important congenital vitreoretinal disorders that can severely affect a child's visual acuity [9]. In three eyes reported here, no retina lesion, macular lesion, retinal breaks and retinal detachment presented. Therefore, we speculated that the complicated cataract may be the main factor resulting in the low visual acuity. A retrospective cohort study analyzed the degree of iris coloboma and the characteristics of the crystalline lens in 56 patients with iris coloboma. They found that various techniques for IOL implantation were selected based on the degree of iris and lens capsule coloboma and these techniques were capable of improving the vision and photophobia of the patients [10]. Khokhar S et al., reported favourable surgical outcomes with phacoemulsification in all densities of cataract with congenital choroidal Coloboma [7]. Jaffe NS et al., reported final visual acuity was excellent in four eyes with congenital colobomata. These four eyes had modern cataract surgery-phacoemulsification with a posterior chamber lens placed in the capsular bag [11]. Reasonably, the surgery of phacoemulsification and intraocular lens implantation was suggested to the two patients and the patients obtained good final vision after surgery and kept the good vision until now.

In conclusion, patients with coloboma of iris and choroid complicated with cataract should have a cataract surgery to improve their $\mathrm{BCVA}$, if there is no retinal detachment.

\section{Compliance with Ethical Standards}

All authors declare that he/she has no conflict of interest. All procedures performed in studies were in accordance with the ethical standards of the Ethics Committee of the Third Affiliated Hospital of the Third Military Medical University and with the 1964 Helsinki declaration and its later amendments or comparable ethical standards. Informed consent was obtained from all individual participants included in the study.

\section{References}

1. Pagon RA (1981) Ocular coloboma. Surv Ophthalmol 25: 223-236.

2. Bard LA (1979) Congenital contractural arachnodactyly and intraocular colobomas. Birth Defects Orig Artic Ser 15: 189-205.

3. Jacobs M, Taylor D (1991) The systemic and genetic significance of congenital optic disc anomalies. Eye (Lond) 5: 470-475. 
4. Jesberg DO, Schepens CL (1961) Retinal detachment associated with coloboma of the choroid. Arch Ophthalmol 65: 163-173.

5. Chen MS, Ho TC, Chang CC, Tsai TH, Hou PK (2007) Retinal detachment in a patient with microphthalmos and choroidal coloboma. J Formos Med Assoc 106: 965-968.

6. Mohamed A, Chaurasia S, Ramappa M, Sangwan VS, Jalali S (2014) Lenticular changes in congenital iridolenticular choroidal coloboma. Am J Ophthalmol 158: 827-830.

7. Khokhar S, Gupta S, Kusumesh R, Kumar G (2013) Outcomes of phacoemulsification in eyes with congenital choroidal coloboma. Graefes Arch Clin Exp Ophthalmol 251: 2489-2490.
8. Gopal L, Badrinath SS, Sharma T, Parikh SN, Shanmugam MS, et al. (1998) Surgical management of retinal detachments related to coloboma of the choroid. Ophthalmology 105: 804-809.

9. Takkar B, Chandra P, Kumar V, Agrawal R (2016) A case of iridofunda coloboma with persistent fetal vasculature and lens subluxation. JAAPOS 20: $180-182$.

10. Li J, Li Y, Hu Z, Kong L (2014) Intraocular lens implantation for patients with coloboma of the iris. Exp Ther Med 7: 1595-1598.

11. Jaffe NS, Clayman HM (1987) Cataract extraction in eyes with congenital colobomata. J Cataract Refract Surg 1987 13: 54-58. 